\title{
sparkTable: Generating Graphical Tables for Websites and Documents with $R$
}

by Alexander Kowarik, Bernhard Meindl and Matthias Templ

\begin{abstract}
Visual analysis of data is important to understand the main characteristics, main trends and relationships in data sets and it can be used to assess the data quality. Using the R package sparkTable, statistical tables holding quantitative information can be enhanced by including spark-type graphs

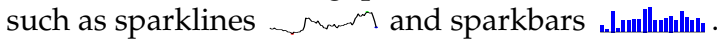

These kind of graphics are well-known in literature and are considered as simple, intense and illustrative graphs that are small enough to fit in a single line. Thus, they can easily enrich tables and texts with additional information in a comprehensive visual way.

The R package sparkTable uses a clean S4 class design and provides methods to create different types of sparkgraphs that can be used in websites, presentations and documents. We also implemented an easy way for non-experts to create highly complex tables. In this case, graphical parameters can be interactively changed, variables can be sorted, graphs can be added and removed in an interactive manner. Thereby it is possible to produce custom-tailored graphical tables - standard tables that are enriched with graphs - that can be displayed in a browser and exported to various formats.
\end{abstract}

\section{Introduction}

Without doubt, visualization of data becomes more and more important for data analysis and for presenting aggregated information for end-users and policy needs. For surveys on the history and developments in visualization we refer to Friendly (2008) and Wickham (2013). The reason for this is that the human eye is a very powerful pattern recognition tool. Readers can easily understand simple visual presentations of data like charts published every day in newspapers, the internet, television and scientific articles. The visual presentation of data should illustrate trends and relationships quickly and easily and should display key figures about data. For readers it is often much easier to understand statistics when seeing a chart or a map rather than being confronted with a long list of numbers (see, e.g., The United Nations Economic Commission for Europe, 2009).

Cleveland and McGill (1987) state that information encoded in a graph depends on a number of aspects such as plotting symbols, length, slopes or colors. Changing any aspect of a graph possibly leads to a different perception and decoding of the information. Thus it is required to research aspects of graphical representation of data and finally come up with recommendations or best practices. This task has been been tackled by various authors (see, e.g., Cleveland and McGill, 1987; Tufte, 1986). Cleveland and McGill (1987) suggest, for example, an optimum mid-angle of 45 degrees when comparing the slopes of different lines. ${ }^{1}$ Other suggestions on how to scale the data exist and are summarized in Heer and Agrawala (2006). Details on the choice of colors can be found in Zeileis et al. (2009).

Tables: Tables are a comprehensive way to summarize the most important facts about complex data. The United Nations Economic Commission for Europe (2009) claims that a small, well-formatted table can provide a great deal of information that readers can quickly absorb, and it, as well as Miller (2004), provide guidelines on how to design tables. However, the possibility of including graphics in tables (and text) is not covered.

Tufte's pioneer work: In our contribution, several examples show how to improve tables by including small graphics in the tables. It is pointed out that an improved table can contain various visual elements. This was originally proposed by Tufte (2001). He introduced the concept of sparklines and their application that is not limited to tables. These intense, simple and word-sized graphics sparklines can be embedded in continuous text and also match with other propositions he has made. For example, they can easily be used together with the small multiples approach (Tufte, 1990). According to Tufte it is always important to answer the question compared to what? when looking at graphs. Thus, the concept of small multiples is simply to put multiple graphs next to each other to allow direct visual comparisons between different (graphical) objects. He also states that small multiples are the best design solution to present a wide range of problems.

\footnotetext{
${ }^{1}$ For example, this is also considered in the sparklines embedded in Table 2.
} 
Another proposition Tufte makes is to show the data above all. In order to do so he introduces the term of data-ink ratio. This ratio can be seen as the ink in a graph used to actually show data by the total ink used to print a graph. While the additional ink used for labels, axes, ticks and grids among others may help to improve graphs, it distracts attention from the most important part of the graph - the data values itself. Thus, in his view the data-ink ratio should be increased whenever possible and sparklines are a way to do so, since it is possible to show a high amount of data, trends and variations in small space. This concept has also been criticized. Inbar et al. (2007) evaluated people's acceptance of the minimalist approach (by increasing the data-ink ratio) to visualize information; a standard bar-graph and a minimalist version (both in Tufte, 2001) were compared. The majority of evaluators was more attracted by the standard graph. However, they didn't evaluate the case of placing sparklines in text or tables. Traditionally, the output in many areas of statistics is focused on tables (The United Nations Economic Commission for Europe, 2009), this is especially true for official statistics. Large tables with lots of number provide a lot of information, but make it hard to grasp it. Visualization can provide tools to make effects in the data visible at first sight and can provide the possibility to show more of the data than by just showing the numbers.

Software to produce sparklines: To make visualization methods applicable, software tools, which provide interactions with the data and provide reproducibility of any result, are needed.

To publish sparklines in newspapers has a long history. For example, the Huntsville Times reports the use of sparklines in their sports section on March 21, 2005. To produce this kind of sparklines and graphical tables, various software products are available. For example, SAS@ and JMP@ include such facilities. ${ }^{2}$ Also Microsoft Excel allows to produce sparklines and there are implementations to produce simple sparklines for Photoshop, Prism, DeltaMaster and matplotlib. Most of these solutions are closed-source and commercial.

\section{The sparkTable package}

Our contribution goes a step beyond the before mentioned available tools. It is not just a reimplementation of sparklines in another software. Various improvements have been made and new features are available to the user.

The presented software is free and open-source, its features are designed in a well-specified, object-oriented manner with defined classes and methods. The presented software allows to save the sparklines in different output formats for easy inclusion in LATEX or websites with many optional graphical features, i.e., the sparklines are highly customizable. The software features the simple generation of graphical tables with any statistical content that is automatically calculated from microdata. Finally, interactive features to create sparklines and sparktables are available and clickable in the browser.

In the following, a short introduction into the R package sparkTable (Kowarik et al., 2015) is given with the help of an example. The first version of the package was uploaded to the Comprehensive $\mathrm{R}$ Archive Network (CRAN) in May 2010 and has been improved various times since then.

The initial task was to find ways how common and frequent output such as tables and reports of statistical organizations could be improved using spark-type graphs and to find a technical solution to create these visualizations. Since $\mathrm{R}$ is gaining momentum in national statistical institutes all over the world, it was a natural choice to create an add-on package for R containing such functionality. There are a variety of reasons for this: one of them is the possibility to easily share and distribute the package and to instantly get feedback from users.

The goal was to create a free and open source software tool that allows the quick creation of spark-type graphics that could easily be included in various documents such as reports or web pages. Many examples for graphical tables have been produced and - based on the feedback from both colleagues and users - it seems as if graphical tables are an helpful tool to improve traditional, existing, tabular-based outputs of national statistical offices. Some of these graphical tables are presented below.

sparkTable can also be used by non experts in R. Interactive clickable features have been added to the package. Especially for graphical tables, some steps of abstraction are still required. However, it has been made easier to customize and change graphical tables by using interactive web applications. Instead of a formal description of classes and methods and internal implementation of the package, the functionality of the package is illustrated with examples.

\footnotetext{
${ }^{2}$ They offer also the link (batch call) to the sparkTable package (Hill, 2011).
} 


\begin{tabular}{|c|c|c|}
\hline Function & Description & Comment \\
\hline $\begin{array}{l}\text { newSparkLine() } \\
\text { newSparkBar() } \\
\text { newSparkBox() } \\
\text { newSparkHist() }\end{array}$ & $\begin{array}{l}\text { creation of sparklines, } \\
\text { sparkbars, sparkboxplots } \\
\text { and sparkhistograms that } \\
\text { may serve as the base for } \\
\text { creating graphical tables }\end{array}$ & $\begin{array}{l}\text { creates objects of } \\
\text { class 'sparkline', } \\
\text { 'sparkbar', 'sparkbox' } \\
\text { and 'sparkhist' }\end{array}$ \\
\hline plot () & $\begin{array}{l}\text { plots objects of class } \\
\text { 'sparkline', 'sparkbar', } \\
\text { 'sparkhist' or 'sparkbox' }\end{array}$ & \\
\hline export() & $\begin{array}{l}\text { saves objects of class } \\
\text { 'sparkline', 'sparkbar', } \\
\text { 'sparkhist', 'sparkbox' or } \\
\text { 'sparkTable' }\end{array}$ & \\
\hline summaryST() & $\begin{array}{l}\text { summary for a data frame } \\
\text { in a graphical table }\end{array}$ & $\begin{array}{l}\text { creates an object of } \\
\text { class 'sparkTable'. Cus- } \\
\text { tomize the output with } \\
\text { setParameter() }\end{array}$ \\
\hline newSparkTable() & $\begin{array}{l}\text { function to create an ob- } \\
\text { ject of class 'sparkTable' }\end{array}$ & \\
\hline $\begin{array}{l}\text { getParameter(), } \\
\text { setParameter() }\end{array}$ & $\begin{array}{l}\text { basic functions to set } \\
\text { parameters for objects } \\
\text { of class 'sparkline', } \\
\text { 'sparkbar', 'sparkbox', } \\
\text { 'sparkTable' } \\
\text { 'geoTable') }\end{array}$ & \\
\hline
\end{tabular}

Table 1: Most important functions of package sparkTable.

\section{Command line usage of sparkTable by example}

The first step is to install and load the package. We assume that $R$ has already been started. Then the package can be simply installed from any CRAN server.

Help files can be accessed from $\mathrm{R}$ by typing help(package = "sparkTable") into the console. This results in a browse-able, linked help archive. Each method, function and data set that comes with the package is documented and also some example code is provided which allows users to become familiar with the package. So as to demonstrate the facilities of sparkTable, example data sets available in the package are used.

Table 1 shows the most important functions and classes of the sparkTable package. Basic functions are available to produce sparklines, e.g., newSparkLine to create an object of class 'sparkline' that can be used as input for the plotting method plot () which dispatches based on the class of the input method. Using method export() it is possible to save mini-plots as files (pdf, png or eps) on the hard-disk. In addition, functions are available to produce graphical tables and calculate summary statistics for tables, see Basic sparks and Graphical tables for practical examples and more explanations.

In the following we show how to create basic spark graphics in Basic sparks. Then we demonstrate how to produce graphical tables that include some sparks in Graphical tables. We finish by introducing the new interactive ways that can be used to change, modify and adjust objects generated with package sparkTable in Interactive features.

\section{Basic sparks}

The data set pop, which is included in the package, is used. This data set contains the Austrian population size for different age groups from 1981 to 2009. We start by loading the data set into the current $\mathrm{R}$ session.

$>\operatorname{data}($ pop, package $=$ "sparkTable") 
This data set - available in long-format - contains three variables. The variable time describes the year, variable variable the different age groups and variable value the corresponding population numbers. Next, an object, which contains all the information required to plot a first sparkline, is generated. This can be achieved using function newSparkLine(). Calling the function and only setting argument values to use the population data of Austria stored in vector pop_ges as shown in the code listing below, we obtain a new object sline (respectively slineIQR, where also the Inter-Quartile-Range (IQR) is visualized) with default settings for a sparkline plot.

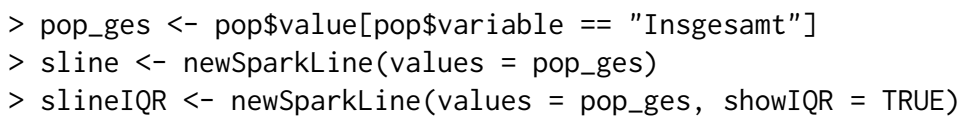

The resulting object sline is of class 'sparkline' and has the default parameter settings. Later it is shown how to change specific settings of the graph using the generic function setParameter(). Note that it is also possible to directly change parameters using newSparkLine () directly. The help-files that can be accessed using ?newSparkLine give an overview on the parameters that can be set or changed.

After creating a sparkline object it is possible to create a plot using function plot() or saving the graphic by applying method export (). Using these methods it is possible to plot and export not only objects of class 'sparkline' but also objects of class 'sparkBar' (generated with newSparkBar()), 'sparkBox' (generated with newSparkBox()) and 'sparkHist' (generated with newSparkHist()). The function export () requires a 'spark*' object as input and allows to specify the desired output format. Currently, one of the following output formats can be selected by changing the function argument outputType:

- "pdf": the spark-graphic is saved in pdf-format;

- "eps": the graph is saved as an encapsulated postscript file;

- "png": the graphic is written to disk as a portable network graph.

The function argument outputType is serialized so that it is possible to create the graphics in all possible formats in one step. By the help of argument filename, users can specify an output filename for the resulting graphic. The code listing below shows how to save the sparkline represented in obj sline as a pdf-graphic with filename first-sl.pdf.

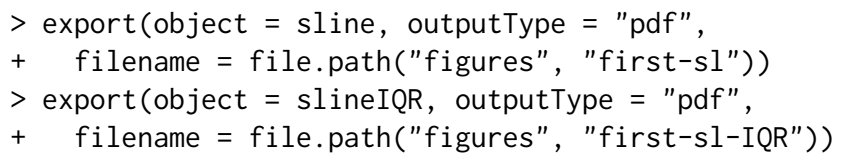

This graph can now easily be included in the current text showing the development__ of the Austrian population from 1981 to 2009, alternatively with integrated IQR

Similarly, other types of graphics can be produced. The next code listing highlights how to create other supported kind of word-graphs. In addition, some of the default parameters are changed.

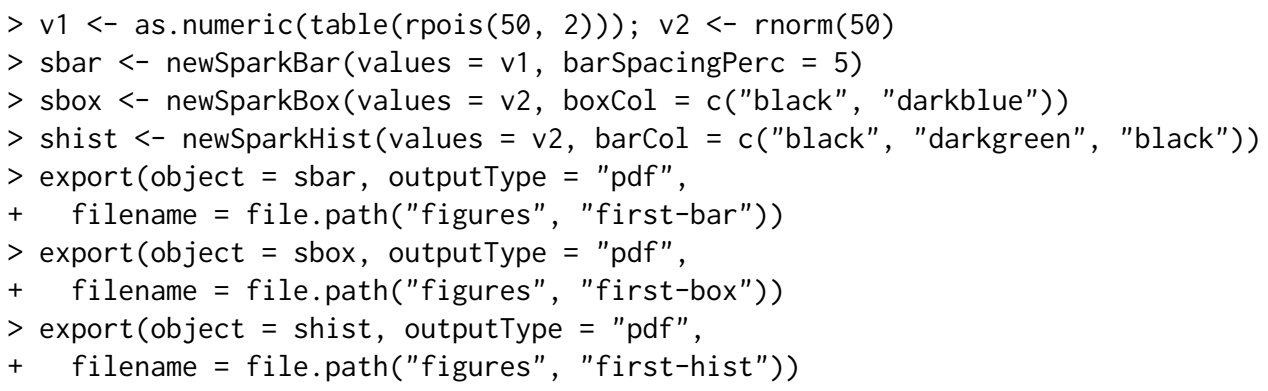

Since they were exported as pdf-files, the produced 1 the _nn sparkhist can be easily integrated into a $\mathrm{LT}_{\mathrm{E}} \mathrm{X}$-document as shown here.

Changing parameters of spark objects can also be achieved by using function setParameter (). In the help file (accessible via ?setParameter) all possible values that can be changed are explained and listed. Some of the options that might be changed are now listed below:

- width: available vertical space of the graph;

- height: available horizontal space of the graph;

- values: data points that should be plotted;

- lineWidth: the thickness of the line in a sparkline-plot; 
- pointWidth: the radius of special points (minimum, maximum, last observation) of a sparkline;

- showIQR: whether the IQR is plotted in a sparkline-graph;

- outCol: color of outlying observations in a sparkbox-graph;

- barSpacingPerc: percent of the total horizontal space used between the bars in a sparkbargraph.

We now show how to change some graphical parameters of the spark graphics we have just produced.

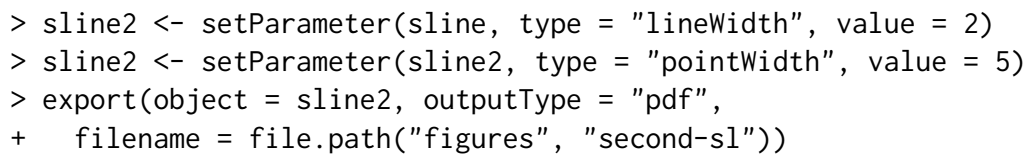

For example the thickness of the line was changed in the sparkline object sline 2 from the default value of 1 to 2 . Additionally, we changed the size of the points for special values in the sparkline to 5 . If this line is plotted, the sparkline showing Austrian population numbers _ looks different.

It is worth noting that the current settings can be queried using function getParameter () in an analogue way as setting parameters by just leaving out the argument values. Detailed information about the kind of information that can be extracted using this function is available from the help page that is accessible via ?getParameter.

Further, it is worth noting that these mini-graphs can also be directly included in fully automated reports. In the following example we show how to include a sparkline into a HTML-document that is created with knitr (Xie, 2014b) and the $R$ markdown language.

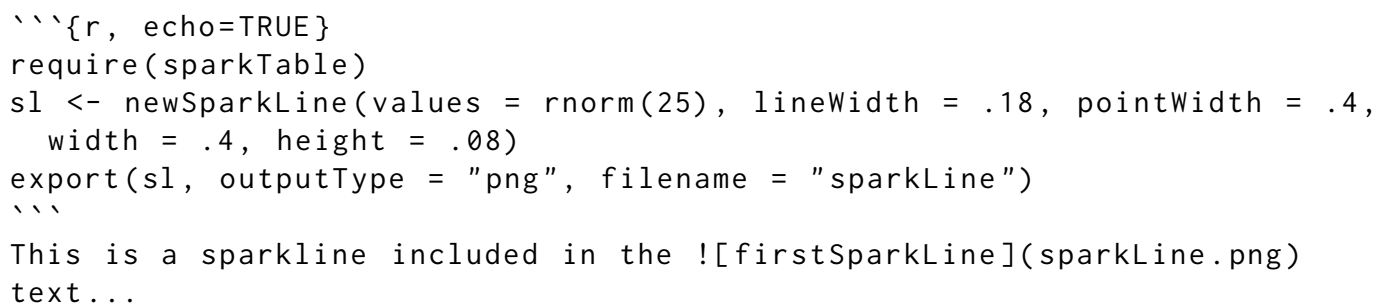

Listing 1: Content of minimal markdown file that contains a sparkline.

A minimal example is shown in Listing 1 . In the first part, a sparkline graph with random values is generated and saved as an image in png-format. We made sure that the height of the image equals approximately the height of a single line. Using markdown code, this image is finally included in the text. The integration in $\mathrm{LT}_{\mathrm{E}} \mathrm{X}$, using Sweave, knitr or brew (Horner, 2011), is straightforward, i.e., the code environment in Listing 1 needs to be changed to Sweave, knitr or brew style.

\section{Graphical tables}

In Basic sparks, the basic usage of the package sparkTable to produce sparklines was demonstrated. Now we show how to enrich tables by including sparkgraphs. In addition, it is shown how sparkgraphs can be used to visualize regional indicators in so-called checkerplots (Templ et al., 2013). We point out that existing tools - specifically xtable (Dahl, 2014) - are used to generate the final output.

In order to plot a graphical table, the first step is to create a suitable input object - in this case an object of class 'sparkTable'. Objects of this kind hold the data itself in a suitable format. Further, such objects contain a list with information about the type of graphs or functions on the data values that should be shown in the resulting table. Moreover, a vector of variable names must be listed to specify for which variables in the data input graphs should be produced. These steps can be achieved using function newSparkTable(). To illustrate this procedure, we give an example using results from the 2013/2014 soccer season in Austria.

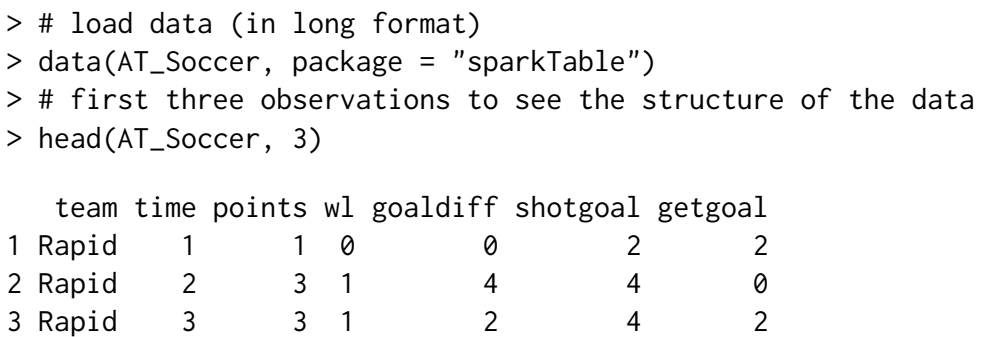




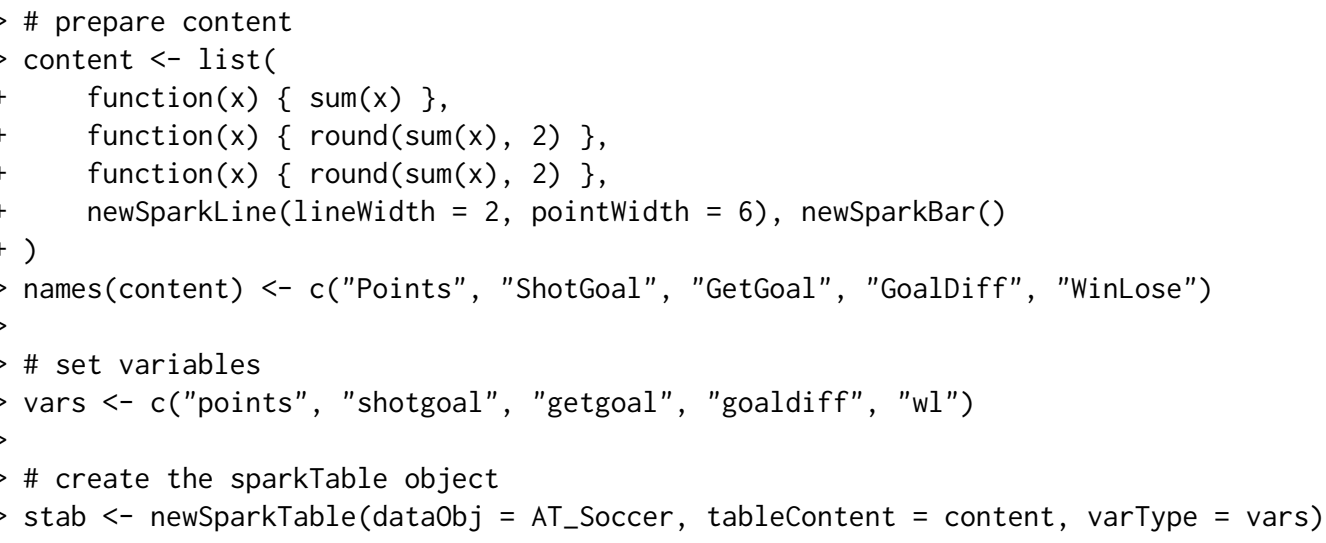

The required code to create a 'sparkTable' object as shown above can be splitted into four sections. In the first line the input data set (in suitable long format for function newSparkTable()) is loaded.

The next step is to prepare a list specifying the content of each column of the required table. In this case the table is specified by five columns. In the first three columns very simple user-defined functions are used to aggregate three different variables. Note that the provided function can be arbitrarily complex.

For the last two columns of the table, sparklines and sparkbars are created. In this case we show that it is also possible to change parameters directly when creating such objects. We used this possibility to change the thickness of the line and the size of points for sparklines as it was already described in Basic sparks. It should also be noted that the names of this input list correspond to column names in the resulting final table, therefore we set the names of this list to the desired column headers. For example, the first column in the final table will have column name Points.

After the object stab has been established, the graphical table can be created. The required code is shown below:

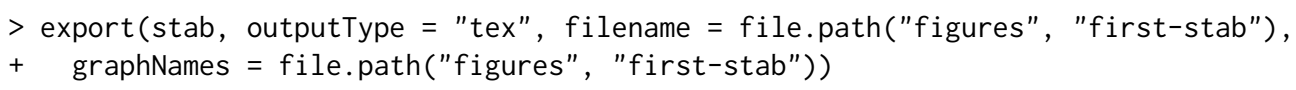

The function export() is applied on the object stab, which has just been created in the previous example. The format of the output can be specified. By setting argument outputType to 'tex', the functions write $\mathrm{LT}_{\mathrm{E}} \mathrm{X}$-code in the file specified with argument filename. Additionally, some instructions on how to include the table into a document are printed in the R session. It is worth to note that the code used to generate the table is also returned to $\mathrm{R}$, which makes it easy to include it into reproducible reports. The final result of this example is shown in Table 2. The embedded sparklines in the second last column of Table 2 present an optimum mid-angle of 45 degrees of the slopes of each time series.

\begin{tabular}{|c|c|c|c|c|c|}
\hline & Points & ShotGoal & GetGoal & GoalDiff & WinLose \\
\hline Rapid & 62 & 63 & 40 & Minn N & 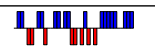 \\
\hline Salzburg & 80 & 110 & 35 & nimpringst & \\
\hline Austria & 53 & 58 & 44 & Vhuthurh & "11" \\
\hline Sturm & 48 & 55 & 56 & wunst. & I"IIIIn|" \\
\hline Groedig & 54 & 68 & 71 & intrypor & minn \\
\hline Neustadt & 39 & 43 & 84 & Mrmw & IIIIIII \\
\hline FC Wacker & 29 & 42 & 70 & midhums & I IIIIIIII \\
\hline Wolfsberg & 41 & 51 & 63 & wunnsis & IIIIIIH \\
\hline Ried & 43 & 55 & 66 & youms & 14in \\
\hline Admira & 42 & 51 & 67 & vompriss & 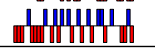 \\
\hline
\end{tabular}

Table 2: A graphical table featuring data values and sparkgraphs applied to Austrian soccer data. For each game in the entire season, wins are maked blue, defeats in read and no bars are plotted for draws. In addition we present differences in goals throughout the season an well as overall points and goals for each team.

In case a user wants to include a table in $\mathrm{AT}_{\mathrm{E} X}$, a short tex-macro ( $\backslash$ graph), which also has to be included in the LTEX source, is printed by default in the R console. This information can be switched off by setting function argument infonote to FALSE. 


\section{Geographical tables}

The concept to create geographical tables is based on the concept of the so called checkerplot (Templ et al., 2013), which is also implemented in the package sparkTable in the function checkerplot(). We now show how to generate a geographical table for the EU using the function newGeoTable() and plotGeoTable(). The final result is shown in Table 3. Let us first load some data and print the first three rows of the data:

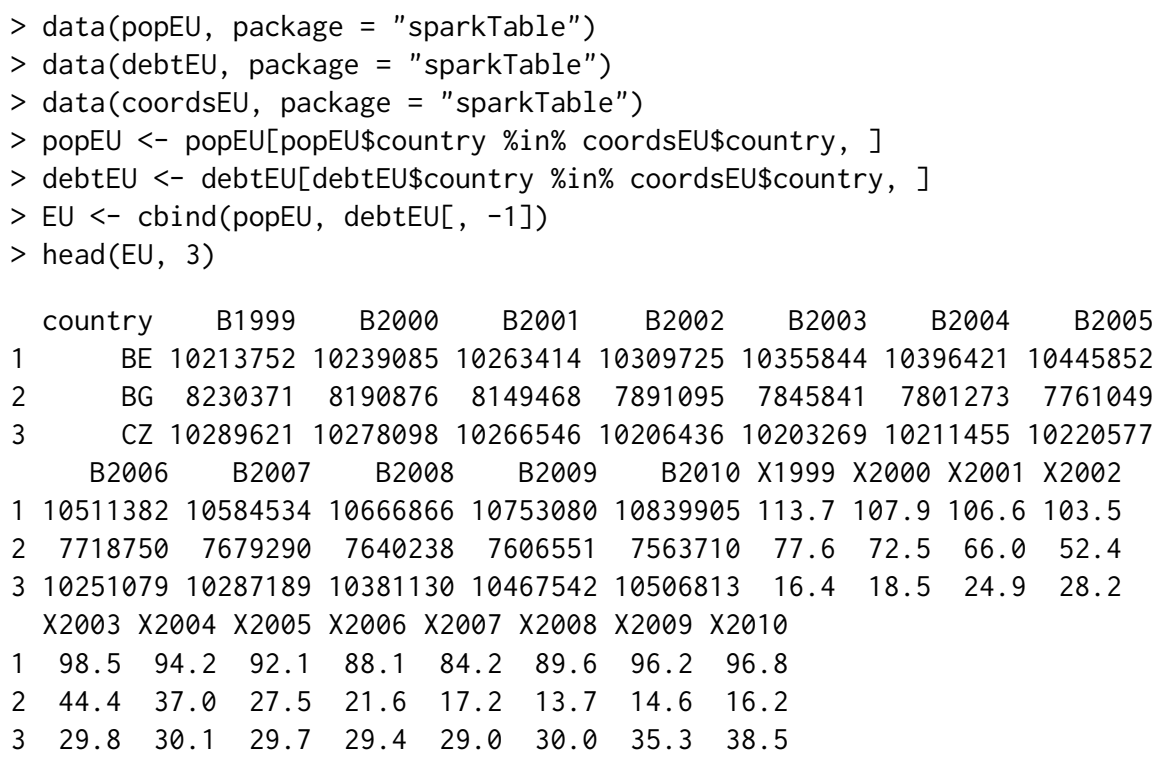

Before we generate sparklines, the data has to be transformed to long format. reshapeExt() can be used to bring the data to the form so that newSparkTable() or newGeoTable() can be applied. In reshapeExt(), parameter idvar defines variables in long format that identify multiple records from the same group/individual and $v$. names corresponds to names of variables in the long format that correspond to multiple variables in the wide format, see ?reshapeExt for details. We show the first two list elements of the reordered data:

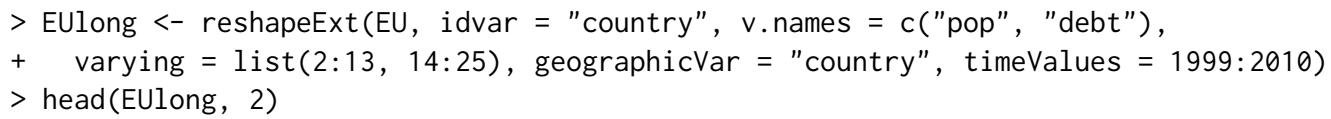

\$BE

$\begin{array}{lrrr} & \text { country time } & \text { pop } & \text { debt } \\ \text { BE.1 } & \text { BE 1999 } & 10213752 & 113.7 \\ \text { BE.2 } & \text { BE 2000 } & 10239085 & 107.9 \\ \text { BE.3 } & \text { BE 2001 } & 10263414 & 106.6 \\ \text { BE.4 } & \text { BE 2002 } & 10309725 & 103.5 \\ \text { BE.5 } & \text { BE 2003 } & 10355844 & 98.5 \\ \text { BE.6 } & \text { BE 2004 } & 10396421 & 94.2 \\ \text { BE.7 } & \text { BE 2005 } & 10445852 & 92.1 \\ \text { BE.8 } & \text { BE 2006 } & 10511382 & 88.1 \\ \text { BE.9 } & \text { BE 2007 } & 10584534 & 84.2 \\ \text { BE.10 } & \text { BE 2008 } & 10666866 & 89.6 \\ \text { BE.11 } & \text { BE 2009 } 10753080 & 96.2 \\ \text { BE.12 } & \text { BE 2010 } & 10839905 & 96.8\end{array}$

$\$ B G$

country time pop debt

BG.1 BG 1999823037177.6

BG.2 BG 2000819087672.5

BG.3 BG 2001814946866.0

BG.4 BG 2002789109552.4

BG.5 BG 2003784584144.4

BG.6 BG 2004780127337.0

BG.7 BG 2005776104927.5

BG. $8 \quad$ BG 2006771875021.6

BG.9 BG 2007767929017.2 


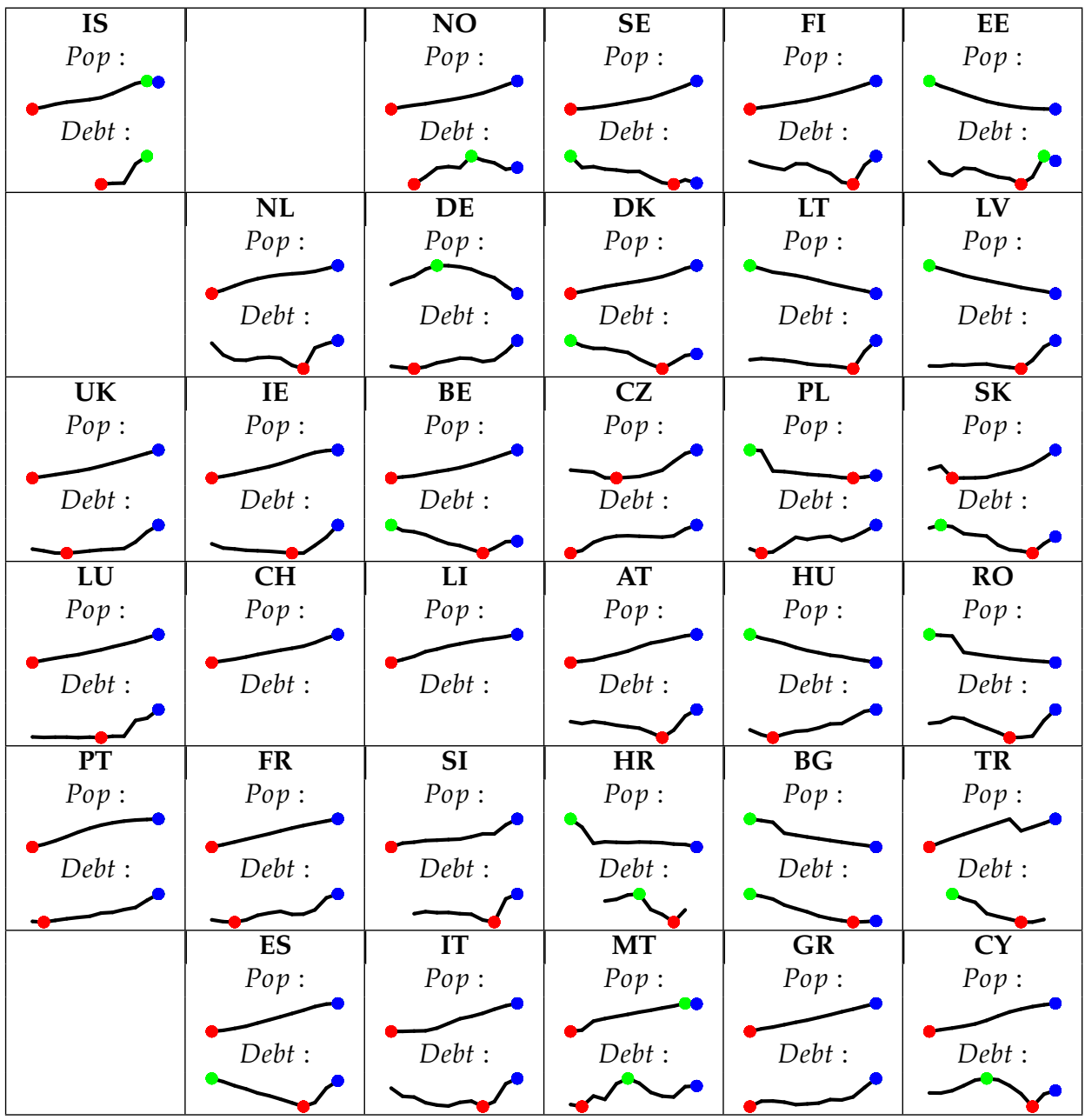

Table 3: A geographical table featuring sparkgraphs for all EU countries.

BG.10 BG 2008764023813.7

BG.11 BG 2009760655114.6

BG.12 BG 2010756371016.2

The data is now ready and we can produce the geographical table:

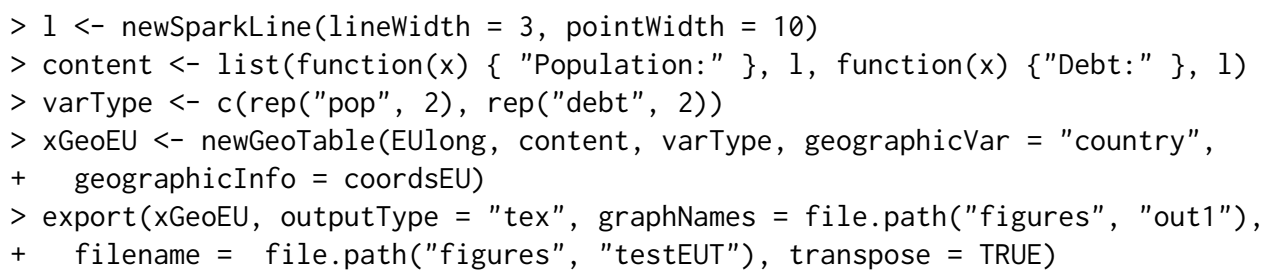

For the geographical representation in a grid, geographical coordinates have to be provided. Internally, an allocation problem is solved by linear programming to find the nearest free grid cell from the provided coordinates under several constraints. For this task, we refer to Templ et al. (2013), resulting in, for example, Scandinavian countries placed in the north, south Mediterranean countries in the south, etc. Again, the sparklines in each grid can be modified by function setParameter().

\section{Interactive features}

Until recently sparkTable only provided rigid views of graphical tables in documents and on homepages. However, when including a graphical table on a homepage some interactivity is beneficial. Two functions using the functionality from shiny (Chang et al., 2015) allow for interactive graphical tables. 
View your graphical table in a shiny app: showSparkTable() is a function to generate a shiny app to view your graphical table directly in a browser with some basic interactive functions like sorting and filtering. At the moment, methods for objects of class 'sparkTable' and 'data. frame' already are implemented. Figure 1 shows the output of the function applied to a 'sparkTable' object.

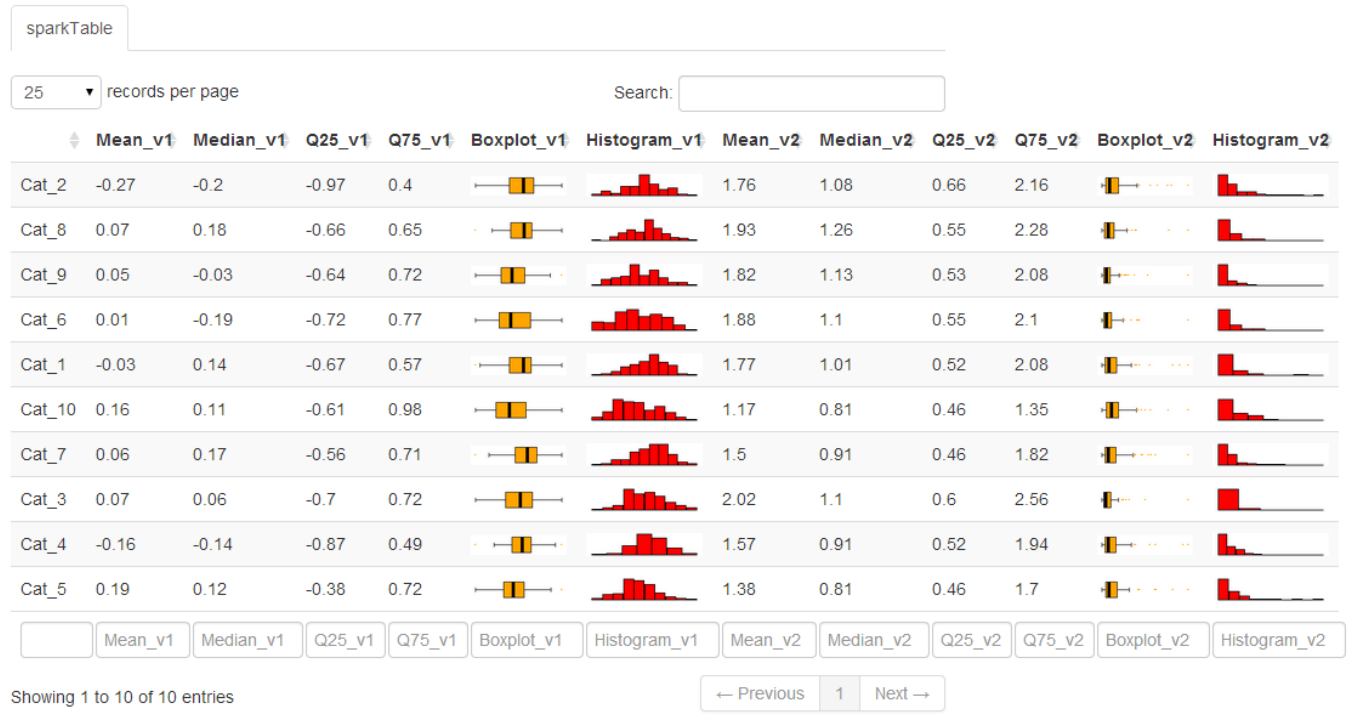

Figure 1: Shiny output of a 'sparkTable' object.

In the following, the key features of customizeSparkTable() are discussed.

\section{Tab 1: Import sparkTable objects}

The provided app is organized in tabs. In each of the five tabs, specific changes can be made. Figure 2 shows the first tab - importData - that is available to the user when the function is called for the first time. We point out that this function has to be started with a 'sparkTable' object as input. In the upper part of this page, all possible groups that are available in the input data object are listed. In the lower part of the page an interactive table is shown. By using the mouse to toggle check-boxes it is possible to choose the groups that should be included in the final output.

\section{Tab 2: Preparation of the table}

The second tab - modify table - that is shown in Figure 3 is also partitioned into two parts. In the upper part, all the columns that are currently present in the output table are listed. It is then possible to select columns that should be deleted. After selecting the corresponding columns by toggling the check boxes and clicking on the remove selected columns-button, these columns are dropped from the table. However, in this tab it is also possible to add an additional column to the table. In this case the user needs to provide a column name and can select both the type of the column and the variable, which should be used, by selecting values from a drop-down menu. All supported types of graphs (sparkline, sparkbar, sparkhist and sparkbox) are available as well as the special type function, which can be used to calculate some statistics of the selected variable. An example is the determination of the maximal data value.

\section{Tab 3: Global options}

In the global options-tab (see Figure 4) it is possible to change the specifics of the current output. For each column, a block of options is listed. The user has the option to change the column name by modifying the current heading in a text box. It is also possible to change the type of a column by selecting a new type from a drop-down box or to adjust graphical parameters (such as point sizes or line width or colors) for some plots. For columns that are of type function, the function definition can be altered. For the adjustment of graphical parameters, suitable inputs such as sliders or drop-down boxes are used. After the desired modification has been applied, the user is required to press the modify-button for the currently changed column. The page will refresh and additional changes can be made. We note, however, that for the time being it is only possible to change one variable after another. 


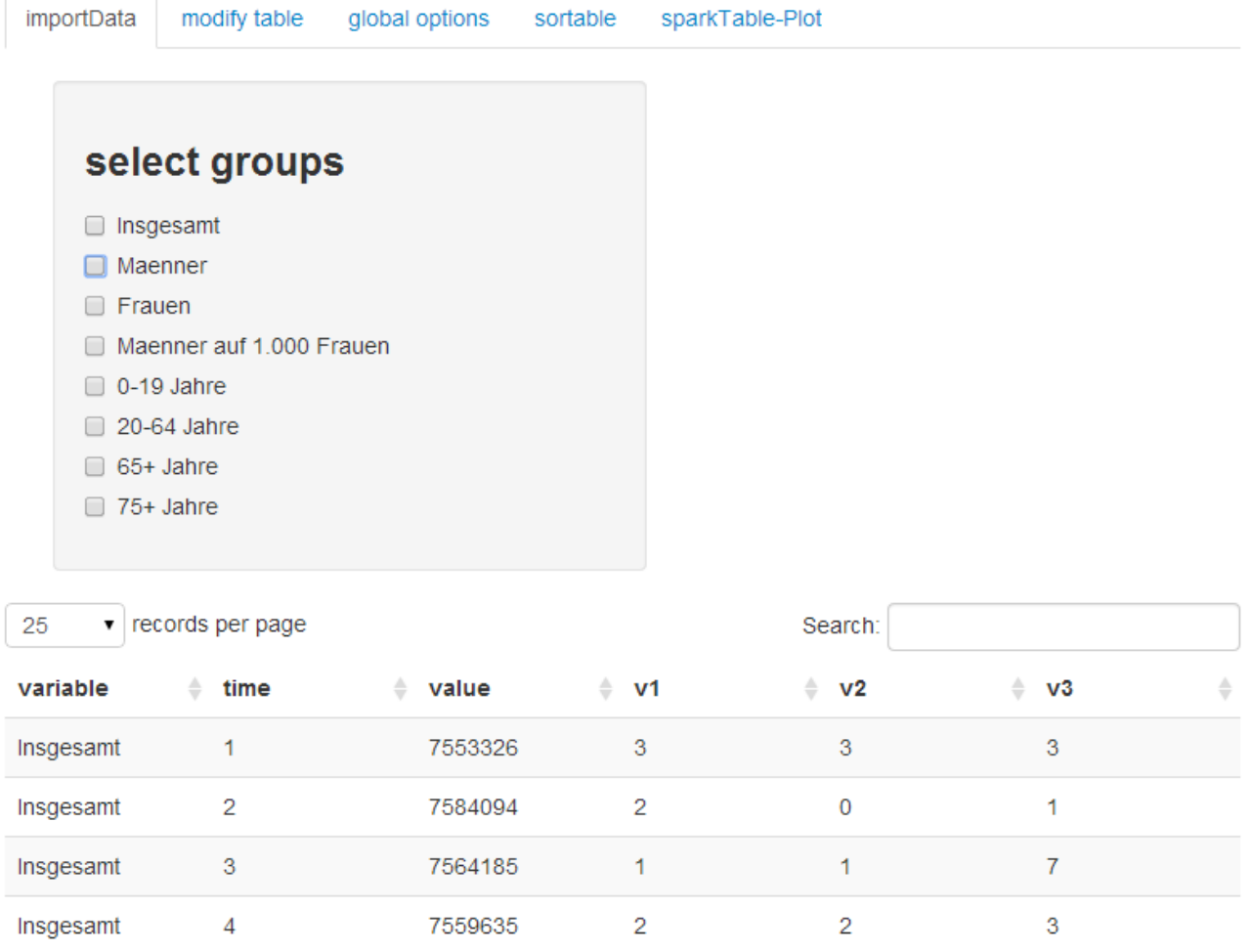

Figure 2: Data options applicable for a 'sparkTable' object.

importData modify table global options sortable sparkTable-Plot

\section{select columns that should be deleted}
$\square$ column1
$\square$ column2
$\square$ column3
$\square$ column 4
$\square$ column 5
$\square$ newcol_52180101769045
remove selected columns

\section{add columns}

column name
newcol_79849867685698

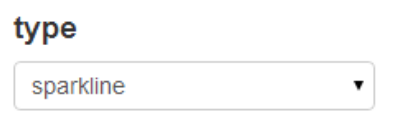

add another column

Figure 3: Possibilities for modifications of the loaded 'sparkTable' object. 


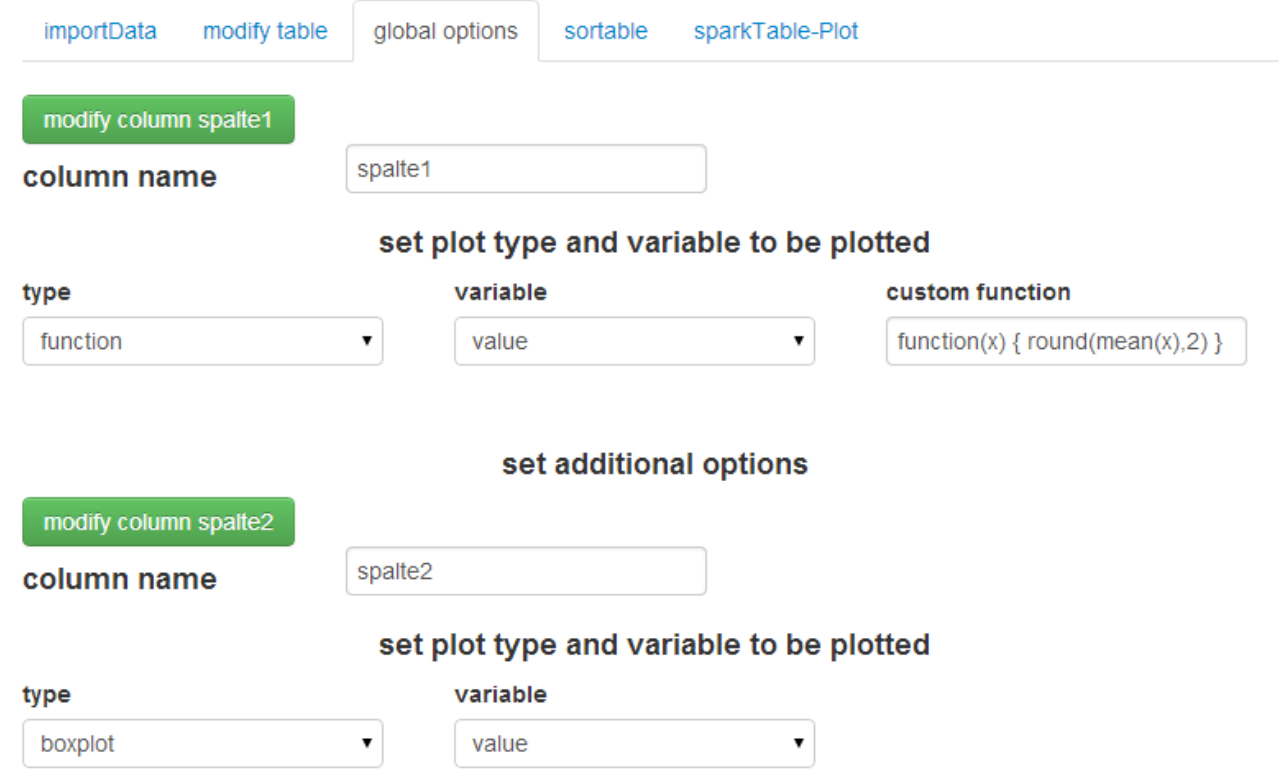

Figure 4: Global options.

\begin{tabular}{ll|l|l} 
importData modify table global options & sortable & sparkTable-Plot
\end{tabular}

\section{please drag and drop the columns into the desired order}

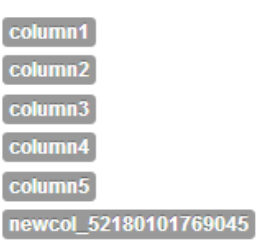

\section{please drag and drop the rows (groups) into the desired order}

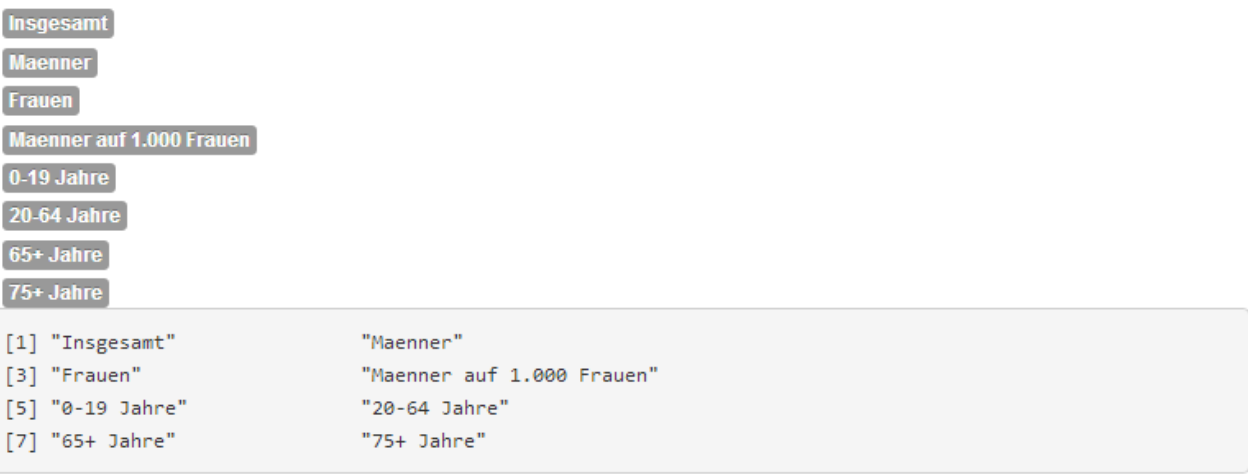

Figure 5: Sort variables and groups in the sparkTable.

\section{Tab 4: Ordering of columns}

In the fourth tab (sortable), which is shown in Figure 5, it is possible to interactively change the ordering of columns (in the first part of the page) and rows (in the second part of the page) by using the mouse to drag boxes around until the desired ordering has been reached. The current result is automatically applied to the 'sparkTable' object, which is always visible in the last tab. 


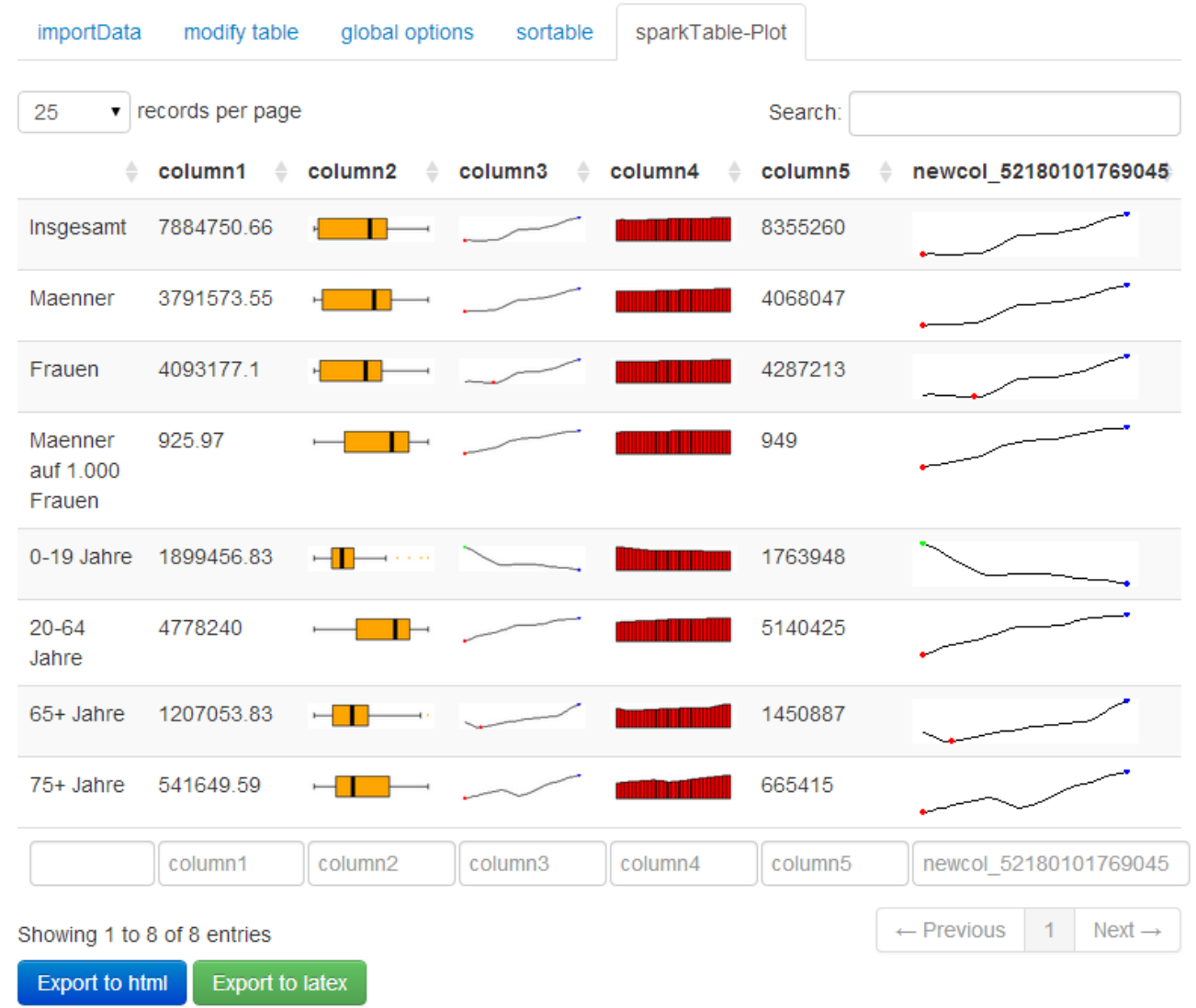

Figure 6: Preview of the output sparkTable.

\section{Tab 5: The graphical table}

In the tab sparkTable-Plot (see Figure 6), the current graphical table with all the current options is displayed. At the bottom of the page the user is offered two buttons. Using Export to html, the graphical table can be exported as an HTML-file, whereas pressing the Export to latex-button exports the final output into tex-format. Furthermore, the required $\mathrm{R}$ code to create the current graphical table is also printed to the current $\mathrm{R}$ session. Thus, users may adjust, copy or reuse a suitable plot-layout that was created with the interactive procedure.

\section{Conclusions}

Eduard R. Tufte's quote "Graphical excellence is that which gives to the viewer the greatest number of ideas in the shortest time with the least ink in the smallest space" holds especially true for graphical tables embedding sparkgraphs, since a lot of information can be put into a table with small but comprehensive (spark) graphs.

We gave a few examples of sparklines and graphical tables, for example, on sparkbars and sparklines in graphical tables to visualize trends and performances in sport results (compare Figure 2). Further obvious applications for sparklines are, e.g., economic and financial data. With the help of such sparklines, it is possible to present graphs, which allow to track and compare trends on changes in time series. Statistical tables can also be enhanced with such types of graphs.

Histograms and box-plots presented in graphical tables can be used to show the distribution of data together with some basic statistics in any publication that present empirical results or analyses in general. Sparkbars can thereby also be used to visualize frequency counts on categories of a variable. For specific summaries of data using sparkboxplots and sparkbars, see for example Hron et al. (2013). Also tabulated information for policy needs can be embedded in graphical tables.

The package sparkTable provides a flexible and interactive way to produce graphical tables for printed or web publication. Graphical tables have the key advantage to provide more information and insight in the same amount of space. A strict class oriented implementation using S4 classes organizes the internal production of graphical tables and sparklines in a pre-defined manner. The users are 
provided with highly customizable functions to create sparklines and tables in a flexible manner. The sparklines can be modified easily and the tables can also contain arbitrarily comples statistics based on the underlying data.

Additionally, interactivity is added with the help of the package shiny. Especially these interactive features allow non-experts in $\mathrm{R}$ to create graphical tables enriched by all kind of sparklines. Tables can then be exported to HTML or LTEX and additionally the code required for various plots is shown both when the interactive shiny-app is used and when graphical tables are exported with export().

\section{Bibliography}

W. Cleveland and R. McGill. Graphical perception: The visual decoding of quantitative information on graphical displays of data. Journal of the Royal Statistical Society. Series A (General), 150(3):192-229, 1987. [p24]

D. B. Dahl. xtable: Export Tables to LATEX or HTML, 2014. URL http://CRAN. R-project. org/package= xtable. R package version 1.7-3. [p28]

M. Friendly. A brief history of data visualization. In C. Chen, W. Härdle, and A. Unwin, editors, Handbook of Data Visualization, pages 15-56. Springer-Verlag, Heidelberg, 2008. [p24]

J. Heer and M. Agrawala. Multi-scale banking to 45 degrees. IEEE Transactions on Visualization and Computer Graphics, 12(5):701-708, Sept. 2006. [p24]

E. Hill. JMP@ 9 add-ins: Taking visualization of SAS@ data to new heights. In SAS Global Forum 2011, 2011. [p25]

J. Horner. brew: Templating Framework for Report Generation, 2011. URL http: //CRAN. R-project. org/ package=brew. R package version 1.0-6. [p28]

K. Hron, M. Templ, and P. Filzmoser. Estimation of a proportion in survey sampling using the logratio approach. Metrika, 76(6):799-818, 2013. [p35]

O. Inbar, N. Tractinsky, and J. Meyer. Minimalism in information visualization: Attitudes towards maximizing the data-ink ratio. In Proceedings of the 14th European Conference on Cognitive Ergonomics: Invent! Explore!, ECCE '07, pages 185-188, New York, NY, USA, 2007. [p25]

A. Kowarik, B. Meindl, and M. Templ. sparkTable: Sparklines and Graphical Tables for $T_{E} X$ and HTML, 2015. URL http: //CRAN. R-project. org/package=sparkTable. R package version 1.0.0. [p25]

J. Miller. The Chicago Guide to Writing about Numbers. Chicago Guides to Writing, Editing, and. University of Chicago Press, 2004. [p24]

RStudio and Inc. shiny: Web Application Framework for R, 2014. URL http://CRAN. R-project.org/ package=shiny. R package version 0.9.1. [p31, 140]

M. Templ, B. Hulliger, A. Kowarik, and K. Fürst. Combining geographical information and traditional plots: The checkerplot. International Journal of Geographical Information Science, 27(4):685-698, 2013. [p28, 30, 31]

The United Nations Economic Commission for Europe. Making Data Meaningful. Part 2: A Guide to Presenting Statistics, 2009. ECE/CES/STAT/NONE/2009/3. [p24, 25]

E. Tufte. Envisioning Information. Graphics Press, 1990. [p24]

E. R. Tufte. The Visual Display of Quantitative Information. Graphics Press, Cheshire, CT, USA, 1986. [p24]

E. R. Tufte. The Visual Display of Quantitative Information. Bertrams, 2nd edition, 2001. [p24, 25]

H. Wickham. Statistical graphics. Encyclopedia of Environmetrics, 2013. Pre-print available at http: //vita.had.co.nz/papers/stat-graphics.html. [p24]

Y. Xie. knitr: A General-Purpose Package for Dynamic Report Generation in R, 2014. URL http: //CRAN. Rproject. org/package=knitr. R package version 1.6. [p28,99]

A. Zeileis, K. Hornik, and P. Murrell. Escaping RGBland: Selecting colors for statistical graphics. Computational Statistics \& Data Analysis, 53(9):3259-3270, 2009. [p24] 
Alexander Kowarik

Statistics Austria

Guglgasse 13; 1110 Vienna

Austria

alexander.kowarik@statistik.gv.at

Bernhard Meindl

Statistics Austria

Guglgasse 13; 1110 Vienna

Austria

bernhard.meindl@statistik.gv. at

Matthias Templ

CSTAT - Computational Statistics

Institute of Statistics \& Mathematical Methods in Economics

Vienna University of Technology \& Statistics Austria

Wiedner Hauptstr. 7; 1040 Vienna

Austria

matthias. templ@tuwien.ac.at 